\title{
The Optimal Steam Pressure of Thermal Power Plant in a Given Load
}

\author{
Yong Hu, Ji-zhen Liu, De-liang Zeng, Wei Wang, Ya-zhe Li \\ North China Electric Power University, State Key Laboratory of Alternate Electrical Power System with Renewable \\ Energy Sources, Beijing, China \\ Email: huyong198610@ncepu.edu.cn
}

Received January, 2013

\begin{abstract}
As the large change of the grid load, many large capacity units of our country had to change the load in order to meet the gird need. When a thermal power plant receives a given load instruction from the grid, it is necessary to set an optimal steam pressure to maintain the high efficiency of the plant. In the past optimization methods, during the process of calculation, the output of the turbine often changed, it was hard to maintain the output constant. Therefore, in combination with the theory of variable condition of turbine, calculation of governing stage and the matrix equation of thermal power system, an optimization method were put forward and an optimal solution was got in a given load.
\end{abstract}

Keywords: Given Load; Pressure Optimization; Variable Condition; Thermal Power Plant

\section{Introduction}

As the development of economy in china, consumption level of the people have enhanced, which leads to a large proportion of electricity power is consumed in our daily life, resulting in the difference between peak and valley of grid load increased year by year. And in our country, large-capacity thermal power plants have a large percentage in the total installed capacity of power plants, which makes the large-capacity units with a basic load have to participate in the load regulation. The units have to deviate from the original design condition and even run in the low load area for a long time, which makes thermal efficiency of the units decrease greatly. Among the factors that affect the thermal efficiency of power plant, only the running modes and operating parameters can be adjusted by operating personnel. Therefore the research of thermal power plants in the off-design condition is of great significance in the selecting of running modes and operating parameters.

In a given load, when the unit runs in a high steam pressure, the ideal enthalpy drop of turbine will increase and the outlet pressure of feed-water pump will rise simultaneously. In order to maintain the given unit load, it is necessary to reduce the steam flow rate through decreasing the opening degree of regulating valves, this will increase the throttling loss of the governing stage. When the unit runs in a low steam pressure, the theoretical thermal efficiency of the unit will reduce, but the lower steam pressure will make the governing stage to maintain higher internal efficiency, and the outlet pressure of feed-water pump will decrease. In order to maintain the unit load, it has to enlarge the opening degree of regulating valves to increase the steam flow rate. Therefore, in tracking of the grid given load, the unit usually deviates from the designed condition, how to select the optimal steam pressure and the running mode has a great influence on the interest of the power plant.

In the traditional method of the pressure optimization, it usually assumed the steam pressure was approximately proportional to the steam flow, when the steam pressure changed, it calculated the steam flow firstly and then calculated the back pressure of governing stage according to the Flugel formula [1], carried on variable condition calculation of the governing stage and the whole turbine. Finally it determined the efficiency of the unit under the changed steam pressure [2]. But in the practical operation of the thermal power plant, it must guarantee the load of the unit equal to the instruction from the grid, when the steam pressure gets higher, it needs to decrease the opening degree of the regulating valves, lower the steam flow to ensure the stability of the load, and vice versa. In the traditional method, due to the approximate proportional relationship between steam pressure and the flow, it leads to the load changed in proportion, not invariable. In some other literatures, in order to ensure the load unchanged, it iteratively calculated the steam flow using the turbine power equation [3], which ignored the characteristic of the governing stage and caused the deviation of the results. 
Therefore, on the base of variable condition calculation method of governing stage and variable condition theory of turbine, using thermal economic matrix equation [4], in order to solve the problems mentioned above, a new calculation method of optimal steam pressure in a given load was put forward, the optimal steam pressure and running modes was got under different loads.

\section{Model of Steam Pressure Optimization}

\subsection{Calculation of the Governing Stage}

In the variable condition calculation of the governing stage, the steam flow through the fully opened regulating valves and the partly opened valve can be expressed as:

$$
\begin{aligned}
G_{n}^{\prime} & =0.648 \cdot \frac{A_{n}^{\prime}}{10 \cdot \sqrt{p_{0} \cdot v_{0}}} \cdot \mu^{\prime} \cdot p_{2} \\
G_{n}^{\prime \prime} & =0.648 \cdot \frac{A_{n}^{\prime \prime}}{10 \cdot \sqrt{p_{0} \cdot v_{0}}} \cdot \mu^{\prime \prime} \cdot p_{2}
\end{aligned}
$$

Then main steam flow rate can be expressed as:

$$
G_{n}=G_{n}^{\prime}+G_{n}^{\prime \prime}
$$

In which $G_{n}^{\prime}$ is the steam flow through the fully opened valves; $G_{n}$ is the steam flow through the partly opened valve; $A_{n}^{\prime}$ is the flow area of fully opened valves; $A_{n}^{\prime \prime}$ is the flow area of partly opened valve; $p_{0}$ is the pressure of main steam; $v_{0}$ is the specific volume of main steam; $\mu^{\prime}, \mu^{\prime \prime}$ is the function of $p_{2} / p_{0}^{\prime}$, $p_{2} / p_{0}^{\prime \prime} ; p_{0}^{\prime}$ is the steam pressure behind fully opened valve; $p_{0}^{\prime}$ is the steam pressure behind partly opened valve; $p_{2}$ is the back pressure of governing stage; $\eta$ is the efficiency of governing stage; $x_{a}$ is the speed ratio of governing stage.

In general, when the steam flows through the fully opened valves, the throttle loss is smaller, so it can be assumed $p_{0}^{\prime}=0.95 \cdot p_{0}$; when the steam flows through the partly opened valve, the opening degree of partly opened valve is $x(x \in[0,1])$, since the annular chamber after the nozzle is in communication with each other, the steam pressure $p_{1}$ behind the nozzle of each nozzle group are the same, the steam pressure behind the unopened regulating valve (i.e., the pressure before the nozzle of unopened valve) is also equal to $p_{1}$. When the opening degree of valve $x$ gradually changes from 0 to 1 , the pressure $p_{0}^{\prime \prime}$ behind partly opened valve will change from $p_{1}$ to $0.95 p_{0}$. In order to facilitate the calculation, $p_{0}^{\prime \prime}=\left(0.95 \cdot p_{0}-p_{1}\right) \cdot x+p_{1}$ is assumed (this assumption is only convenient to calculate $p_{0}^{\prime \prime}$, it has no effect on the optimization results). So when the opening degree of all the regulating valves is known, the main steam flow can be expressed as:

$$
G_{n}=f\left(p_{0}, x, p_{2}\right)
$$

Therefore, the main steam flow can be determined by $p_{0}, x$ and $p_{2}$, then on basis of the variable condition calculation of governing stage, the steam enthalpy of governing stage $h_{t j}$ can be got.

\subsection{Calculation of the Intermediate Stage and Last Stage}

In the variable condition calculation of turbine, because the flow area of intermediate stage is constant, when the load of the unit is changed, if the variation of temperature before all stages is ignored, the pressure before intermediate stage is proportional to the steam flow of this stage, so pressure ratio is invariant, the efficiency of intermediate stage is unchanged, the ideal enthalpy drop of each stage is also unchanged [5]. Therefore, when the parameters of governing stage are known, the steam enthalpy of each extraction point can be expressed as:

$$
h_{(i+1) 1}=h_{i 1}-\left(h_{i 0}-h_{(i+1) 0}\right)
$$

In which $h_{i}$ is the steam enthalpy of ith stage; subscript 0 represents the designed condition; subscript 1 represents the variable condition.

For the last stage of steam turbine, we calculated from the last stage to the prior stage, found the superheated steam extraction point and set it as ith stage. The steam after the ith stage does adiabatic expansion in the turbine, so the entropy is constant. Combined with the steam pressure of extraction point, the ideal steam enthalpy of this stage could be got, according to (6), we could get the steam enthalpy of this stage and calculated one stage by one stage until to the last stage.

$$
h_{(i+1) 1}=h_{i 1}-\eta_{i, i+1}\left(h_{i 1}-\tilde{h}_{(i+1) 1}\right)
$$

In which $\tilde{h}_{(i+1) 1}$ is the ideal steam enthalpy of $(i+1)$ stage, $\eta_{i, i+1}$ is the efficiency of $(i+1)$ stage.

\subsection{Calculation of the Boiler Feed-Water Pump Turbine}

When the main steam pressure and flow rate change, the output of Boiler Feed-Water Pump Turbine (BFPT) will change too. Therefore, the influence of BFPT on the thermal efficiency cannot be ignored.

From the outlet of feed-water pump to the main steam valve, the phase of working fluid changed. In this process, there exists the loss of resistance along the way and the loss of local resistance [5], both loss can be expressed as:

$$
\Delta p=\zeta \cdot \rho \cdot \frac{C^{2}}{2}
$$

In which $\Delta p$ is the pressure drop; $\rho$ is the average density of fluid; $C$ is the flow rate of fluid; $\zeta$ is the loss coefficient which depends on the characteristic of pipe. We use subscript $d$ represent the parameters of 
design-condition, then the outlet pressure of feed-water pump can be expressed as:

$$
p_{p 2}=p_{0}+\left(\frac{G_{n}}{G_{n d}}\right)^{2} \cdot \frac{\rho_{d}}{\rho} \cdot\left(p_{p 2 d}-p_{0 d}\right)
$$

In which $p_{p 2}$ is the outlet pressure of feed-water pump; $p_{0}$ is the main steam pressure; $G_{n}$ is the main steam flow. When the feed-water flows through the pump, the pressure of feed-water will rise because of the working of pump, this will make the feed-water enthalpy rise. This process can be regarded as isentropic flow [6], so the enthalpy- rise of feed-water can be expressed as:

$$
\Delta h=\frac{\bar{v} \cdot\left(p_{p 2}-p_{p 1}\right)}{\eta_{p}}
$$

In which $p_{p 1}$ is the inlet pressure of feed-water pump; $\bar{v}$ is the average specific volume of feed-water; $\eta_{p}$ is the efficiency of feed-water pump. According to the law of conservation of energy, the extraction flow for BFPT can be got.

$$
D_{B F P T}=\frac{G_{n} \cdot\left(p_{p 2}-p_{p 1}\right) \cdot \bar{v}}{\left(h_{4}-h_{p c}\right) \cdot \eta_{p} \cdot \eta_{j}}
$$

In which $h_{4}$ is the inlet steam enthalpy of BFPT; $h_{p c}$ is the exhaust enthalpy of BFPT; $\eta_{j}$ is the efficiency of BFPT.

\section{Optimization Method of Steam Pressure in a Given Load}

\subsection{Optimization Method}

In order to maintain the output of the unit and overcome the shortcomings of traditional optimization methods, in the process of optimization, we adopted the sequential calculation method, combining with assumption, verification and iterative adjustment. If the load and an initial steam pressure were given, we could get the steam flow, opening degrees of regulating valves, back pressure of governing stage and thermal efficiency of the unit, then a unique mapping relationship was formed among them.

Step1. According to the load instruction, use Figure 1 to determine the feasible range of steam pressure [7] and the number of fully opened valves.

Step2. Assume a certain back pressure of governing stage and the degree of partly opened valve to determine the main steam flow, the enthalpy and temperature of governing stage.

Step3. According to the enthalpy and temperature of the governing stage, carry on the variable condition calculation of intermediate stage and last stage, get the output of turbine.

Step4. Judge the parameters of governing stage using (11). Equation (11) is the Flugel formula [1]. If the equation does not hold, adjust the back pressure of governing stage, and then go to step 2 .

$$
\frac{G_{n}}{G_{n d}}=\sqrt{\frac{p_{2}^{2}-p_{c}^{2}}{p_{2 d}^{2}-p_{c d}^{2}}} \cdot \sqrt{\frac{T_{2 d}}{T_{2}}}
$$

Step5. Judge the output of turbine. If the output of turbine is not equal to the load instruction, adjust the degree of partly opened valve and go to step 2 .

The flow chart of the pressure calculation is shown in

\section{Figure 2.}

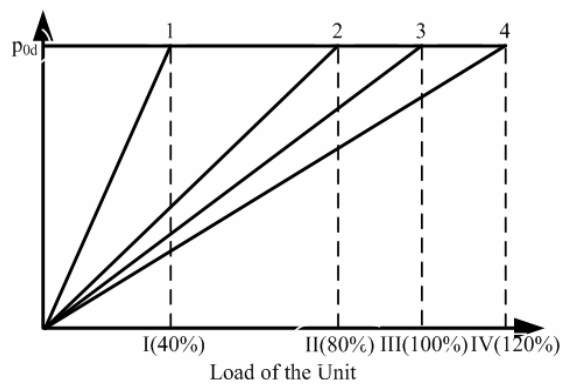

Figure 1. The feasible range of steam pressure.

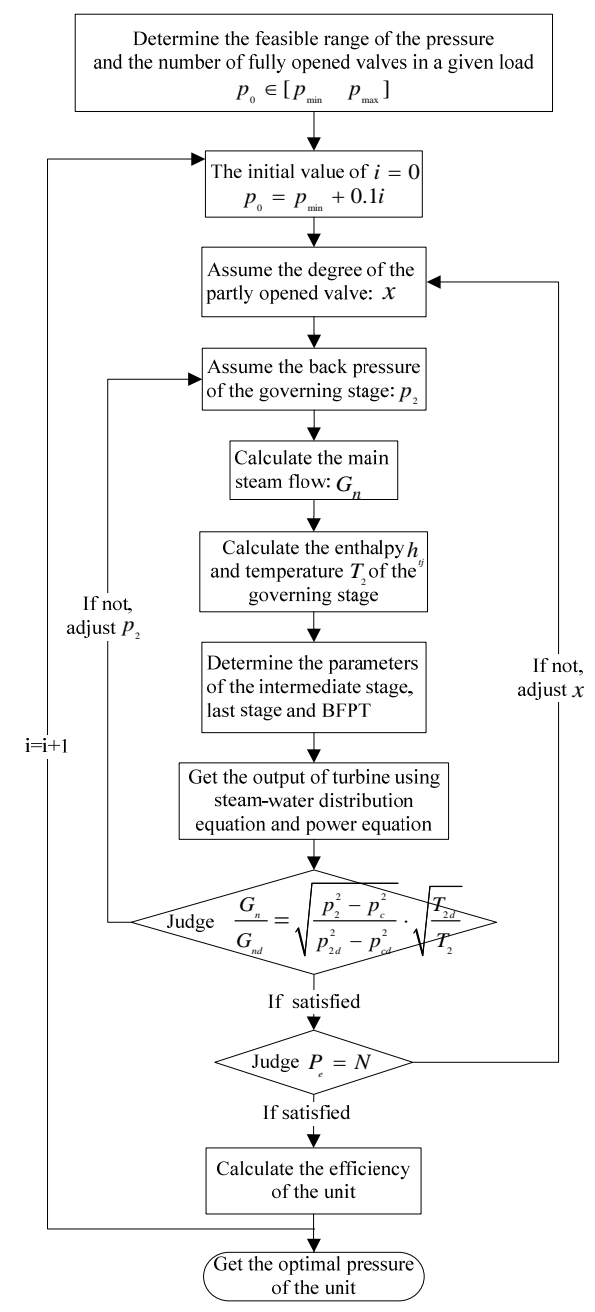

Figure 2. The flow chart of the optimization method. 


\subsection{Application Examples}

We took the Oriental steam turbine N1000-25.0/600/600 as an example, the impact of the overlap of regulating valves was not considered and we ignored the influence of environmental factors on the thermal economy of the unit. In the ideal condition of $100 \%$ load, there were three regulating valves fully opened and one valve closed.

First, we took the $100 \%$ THA condition as an example, analyze and validate the optimization method, the results were shown in Table 1. In the 100\% THA condition, in order to maintain the unit load, as the decline of main steam pressure, the regulating valves had to be opened larger to increase the main steam flow, and the power consumed by feed-water pump was decrease too. The thermal efficiency of the unit was decline as the steam pressure became lower. But when the main steam pressure reduced to 22.76 Mpa, four regulating valves were all fully opened, the throttling losses was least at this moment, so the efficiency of the unit rebounded a little. From the dates of Table 1, the variation tendency of pressure and efficiency consistent with the theoretical analysis, so this method can be used to optimize other conditions of the unit.

Figure 3 shows the thermal efficiency change as the number of fully opened valves change from 2 to 4 in different load. As the increasing of the opening degree, the

Table 1. The analysis of efficiency in a design condition.

\begin{tabular}{ccccc}
\hline $\begin{array}{c}\text { Steam } \\
\text { Pressure } \\
\text { (Mpa) }\end{array}$ & $\begin{array}{c}\text { Main } \\
\text { Steam }\end{array}$ & $\begin{array}{c}\text { Degree of } \\
\text { Regulating } \\
\text { Valves }\end{array}$ & $\begin{array}{c}\text { Thermal } \\
\text { Efficiency }\end{array}$ & $\begin{array}{c}\text { The Energy } \\
\text { Consumption of } \\
\text { Feed-water Pump (MW) }\end{array}$ \\
\hline 25.00 & 2850.95 & $75.00 \%$ & $49.0875 \%$ & 34.6134 \\
24.00 & 2880.56 & $79.25 \%$ & $48.8659 \%$ & 33.7388 \\
23.60 & 2885.50 & $83.75 \%$ & $48.8319 \%$ & 33.2834 \\
23.20 & 2889.22 & $90.50 \%$ & $48.8106 \%$ & 32.8091 \\
23.00 & 2889.43 & $94.50 \%$ & $48.8095 \%$ & 32.5484 \\
22.76 & 2888.78 & $100.0 \%$ & $48.8159 \%$ & 32.2231 \\
\hline
\end{tabular}

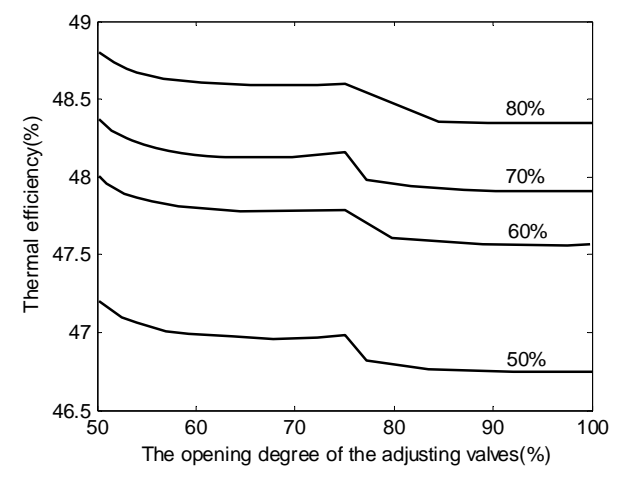

Figure 3. The relation between thermal efficiency and valve opening.

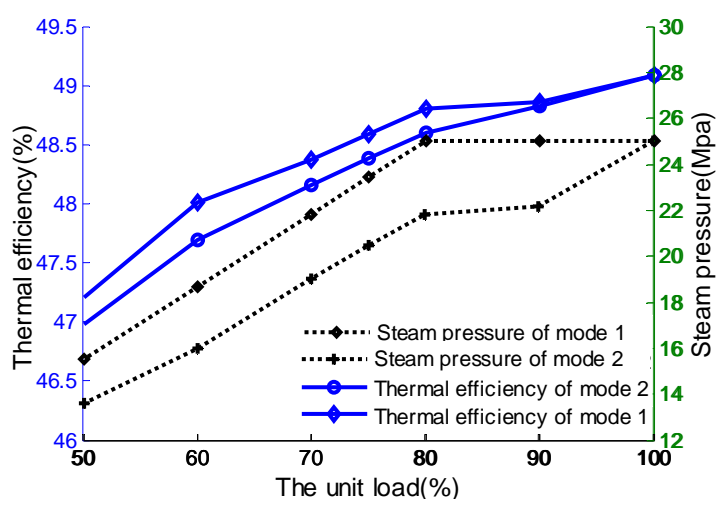

Figure 4. The comparison curves of the two modes.

main steam pressure dropped, the thermal efficiency declined, but in the fully opened points, there existed a local optimal point.

Figure 4 shows the efficiency of the unit in different sliding pressure operation mode. In mode 1 , the unit took a fixed pressure operation mode with 25Mpa steam pressure first, when the output of the load decreased to the $80 \%$, the unit took the sliding pressure operation mode with 2 regulating valves fully opened. In mode 2, the unit took the sliding pressure operation mode with 3 regulating valves fully opened beginning from the $100 \%$ THA condition. It can be seen from the picture, the efficiency of mode 1 was higher than mode 2, especially in the low load region.

\section{Conclusions}

Basing on the variable condition calculation method of governing stage and variable conditions theory of turbine, a new optimization method was put forward for the optimal operation of thermal power plant, and we took the Oriental steam turbine as an example, got the optimal steam pressure of different load and the optimal sliding pressure curve. Therefore, operating personnel can adopt this method, combined with the characteristic of the unit and the factor of environment, drawing the optimal pressure curves, which can be used as a reference in the practical operation.

\section{Acknowledgements}

This work was supported by the National Basic Research Program of China (“973” Project) (Grant No. 2012CB215203) and the National Natural Science Major Fund Project (Grant No. 51036002)

\section{REFERENCES}

[1] C. F. Zhang and Y. H. Cui, "The Distinguishing Theory of Critical State of Turbine and Improved Flugel Formula," Science in china Series E, Vol. 33, No. 3, 2003, pp. 
264-272.

[2] L. X. Zhou and M. Hua, "Method for Calculating Main Steam Pressure and Heat Rate Correction Curves Under Off-design Operating Conditions," Journal of Engineering for Thermal Energy and Power, Vol. 26, No. 3, 2011, pp. 351-353.

[3] Z. P. Yang and Y. P. Yang, "Sensitivity Analysis on Energy Consumption of Exhaust Steam Pressure of 1000 MW Steam Turbine Unit, " East China Electric Power, Vol. 39, No. 2, 2011, pp. 2064-2067

[4] S. L. Yan and C. F. Zhang, "The Steam-Water Distribution General Matrix Equation of Thermal System for the Coal-Fired Power Unit," Proceedings of the CSEE, Vol.
20, No. 8, 2000, pp.69-73.

[5] N. Zhao, "The Research of the Relation between Pressure Rate and Flow and the Target Value of the Thermal Parameters under Variable Working Conditions of Steam Turbine,” North China Electric Power University, 2008.

[6] P. Li and M. Hua, "Research on Target Value of BFPT Parameters for Sliding Pressure Operation Unit,” Thermal Turbine, Vol. 39, No. 4, 2010, pp. 251-254.

[7] C. F. Zhang and H. J. Wang, "Quantitative Research of Optimal Initial Operation Pressure for the Coal-fired Power Unit Plant," Proceedings of the CSEE, Vol. 26, No. 4, 2006, pp. 36-40. 